\title{
Investigating dose response relations in occupational mortality studies: something to keep in mind
}

\author{
G M H SWAEN, ${ }^{1}$ A VOLOVICS ${ }^{2}$ \\ From the Departments of Occupational Medicine, ${ }^{1}$ and Biostatistics, ${ }^{2}$ State University of Limburg, \\ PO Box 616, 6200 MD Maastricht, The Netherlands
}

Many epidemiological studies assessing the long term health effects of occupational (or non-occupational) exposures have used the design of a non-concurrent prospective or cohort study. ${ }^{12}$ In this type of study a group of workers, exposed in the past, is identified and followed up through time to determine the age and time specific mortality rates for several different causes of death.

The statistical analysis usually consists of a persontime analysis, resulting in a standardised mortality ratio (SMR). The purpose of applying the person-time analysis in cohort studies is to adjust for the influence of the specific age distribution of the population under investigation and the changing background mortality rates over time. A person-time analysis is a simple statistical procedure in which the person-years of follow up are specified for each age group and period. The person-years in each cell are multiplied by the death rates of the general population for that particular age group and period giving an "expected number of deaths." The sum of the expected numbers for all the cells combined is the number of expected deaths in the total group. The SMR is calculated by dividing the observed number of deaths by the expected number, multiplied by 100 .

\section{Dose response relations}

By analogy with experimental study designs, epidemiologists have often tried to quantify dose response relations since the existence of a dose response relation is considered to be strong evidence for an actual causal association. ${ }^{3}$ Since the actual level of exposure experienced by the individual study subjects is not usually known, another quantitative measure of exposure is often chosen. This quantitative measure of exposure is duration of employment. One of the underlying assumptions is that the longer a person has worked in a particular occupation the higher the dose he has experienced. Based on this assumption specific dose groups may be distinguished by defining subgroups of workers by duration of exposure which usually coincides with duration of employment. Aftez having defined various dose groups an obvious way to compare the risks of dying from a particular disease iso to calculate the cause specific SMRs for each particuos lar group. The way to do this is to regard the particu $N$ lar dose groups as independent groups and to conduct? a person-time analysis for each separate dose group. The examples of published cohort studies in whicks analyses of this type of dose response relations are described are abundant. Unfortunately, these papers seldom contain information on how exactly the analo ysis of dose response was carried out. Therefore on $\mathrm{e}^{\mathrm{e}}$ cannot check how the SMRs for each particular dgse् group were calculated.

\section{AN EXAMPLE}

An example may help clarify the issue. Suppose a co hort study has been conducted on a group of 10 work ers (A to $J$ ) who all started working in a particulag factory in 1960. They have been followed up untit 1980. Two workers (A and B) died in 1963, one (CP died in 1968, and two (D and $E$ ) in 1975. Two workers ( $F$ and $G$ ) left the company in 1963 and were alive af the end of the study. The remaining three $(\mathrm{H}, \mathrm{I}$, and $\mathrm{J}$ were still employed in the factory in 1980 . The person years of the individual workers are displayed in fig 13

The total study group may be divided into two sub groups, having less than 10 years of exposure or more than 10 years of exposure. Subjects A, B, C, F, and $\mathcal{O S}_{3}$ fall into the subgroup with less than 10 years exposure. The other workers have all experienced more than 10 years exposure. The number of person-years in the short exposure group add up to 54 and in the long exposure group to 90 , including the first 10 years under observation.

\section{HANDLING THE PERSON-YEARS UNDER}

OBSERVATION

The hypothetical cohort study presented above and the division of study subjects into two exposurø groups is the generally accepted way to evaluate the existence of dose response relations. It must be cleart 
Investigating dose response relations in occupational mortality studies something to keep in mind

O Person-years counted in short exposure group. Person-years counted in long exposure group.

Accumulated person-years

In short exposure In long exposure Total group group
A OOO+
B $000+$
c $00000000+$

3

3

8

D $000000000000000+$

15

E $000000000000000+$

F 00000000000000000000

20

G 00000000000000000000

H 00000000000000000000

20

20

100000000000000000000

20

J 00000000000000000000

20

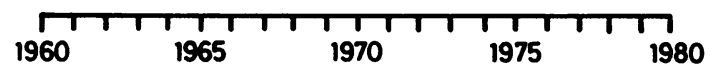

54

90

144

Fig 1 Person-years of 10 workers enrolled in a hypothetical cohort study.

Person-years counted in short exposure group.
Person-years counted in long exposure group.

A $000+$

B OOOt

C $00000000+$

D $000000000000000+$

E $000000000000000+$

F 00000000000000000000

G 00000000000000000000

H 00000000000000000000

I 0000000000000000000

J 00000000000000000000

$1960{ }_{1965}^{T}{ }_{1970}^{T}{ }_{1975}^{T} T_{1980}$

Accumulated person-years

In short exposure In long exposure Total group group
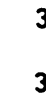

Fig 2 Person-years of 10 workers enrolled in a hypothetical study by exposure group in which the person-years are counted. 
however, that the two workers who died after three years of exposure (A and B) were forced into the short exposure group as a result of the independent or outcome variable of the study. On the other hand, in the first 10 person-years of the workers in the $10+$ exposure group no deaths can have occurred, since if death had occurred the worker would have been categorised in the short exposure group. Counting the deaths in the short exposure group and the living in the long exposure group will artifically result in a higher SMR in the short exposure group and a lower SMR in the long exposure group. One alternative to deal with this problem would be to disregard those person-years of observation before the moment that enough exposure time could have been accumulated to be entered into the long exposure group. In this way events related to the study outcome (mortality) will not influence the subdivision into the two exposure groups. The person-years of all the workers accumulated in the first 10 years of observation, however, should be added into the short exposure group. Then after the first 10 years of observation, actual person-years at risk may be accumulated by those workers who have been exposed for more than 10 years. The first 10 years of observation even for those who are categorised into the long exposure group must be counted in the low exposure group since they were at risk of dying and ending up in the low exposure group. The proposed analysis of the personyears is presented in fig 2 .

By handling the data in this way workers switch to the long exposure group after actually having accumulated 10 years exposure. The scope of this article is limited to handling simple dose response relation and other issues, such as latency, have not been considered.

\section{Conclusion}

Retrospective cohort studies of occupational groups have greatly expanded the knowledge about the long term health effects of the occupational environment. $\overline{2}$ One subanalysis that is generally conducted in these ${ }_{\circ}^{\mathbb{D}}$ types of studies is the investigation of dose response relations. Readily available computer programs haves? facilitated the conduct of the person-time analysis.0 These programs may also be used to calculate SMRso for particular subgroups-for example, dose group $\frac{\bar{s}}{1}$ defined by duration of employment. If these dose $\Omega$ groups are analysed in this way an error may ben made. It must be kept in mind that the subgroups ares not independent. One correct way to handle this is to? add all person-years into the short duration group $\vec{\overrightarrow{ }}$ that were accumulated before achieving the eligibilityo criterion of the long duration group. We also recommend authors of retrospective cohort studies to pro-3 vide a detailed description of how the statistical analysis was conducted, in particular the analysis of dose response relations. We have found one article in which this was done and from which we could con $\stackrel{+}{\mathrm{N}}$ clude that the analysis of the dose-response relation was handled correctly. ${ }^{4}$

Finally, we suggest that epidemiologists who wish pD $^{\circ}$ to publish results of cohort studies and intend to present dose response relations provide more infor $-\frac{\mathbb{D}}{3}$ mation about the procedures conducted during the calculation of these dose response relations.

\section{References}

1 Cole P, Goldman MB. Occupation. In: Fraumeni JF, ed Persons at high risk of cancer. New York: Academic Press, 1975.

2 Decoufle P. Occupation. In: Schottenfeld D, Fraumeni JF, eds. Cancer epidemiology and prevention. London: $\mathrm{W}$ Saunders Company, 1982.

3 Lilienfeld A, Lilienfeld DE. Foundations of epidemiology. New York: Oxford University Press, 1980:309.

4 Buffer PA, Wood S, Eifler C, Suarez L, Kilian DJ. Mortality experience of workers in a vinylchloride monomer production plant. J Occup Med 1979;21:195-203. 